\title{
La place des établissements scolaires en France sous la Ve République: une recomposition parallèle des formes de la justice et des formes de l'État (1959-2009)
}

\author{
Jean Louis Derouet \\ Université Lumière Lyon 2, França
}

\begin{abstract}
Résumé
L'article propose une étude du rôle et de la place des établissements scolaires dans les trois dernières Lois régissant le système éducatif français. Son but est de montrer que ceux-ci correspondent à une recomposition parallèle des formes de la justice et des formes de l'Etat. La Loi de modernisation du système éducatif de 1975 tentait de mettre en œuvre un objectif d'égalité des chances correspondant à l'Etat-providence dans une organisation centralisée et même autoritaire. La Loi d'orientation de l'éducation de 1989 a poursuivi le même objectif d'égalité mais l'a mis en œuvre dans un système déconcentré dont la régulation reposait sur un contrat entre le projet des familles et le projet des établissements. La Loi d'orientation et de programmation de 2005 reprend ce souci de respect des droits des usagers et introduit une obligation de résultats correspondant à une conception managériale de l'Etat.
\end{abstract}

Mots-clé

Politiques d'éducation; Justice; Établissements scolaires; Sociologie de l'Etat

Le sens ordinaire de la justice concernant l'éducation et la formation a considérablement évolué au cours des trente dernières années dans les sociétés atlantiques. Celles-ci avaient hérité des Lumières une conception qui 
présentait l'objectif d'égalité comme la définition moderne de la justice. Cette définition avait nourri la construction de l'État-providence pour qui l'école constituait un des instruments essentiels de son projet de redistribution. Cet ensemble a abouti au programme éducatif de la première modernité, mis en forme par l'OCDE après la Seconde Guerre mondiale: un idéal d'égalité des chances; une école compréhensive pour tous jusqu'à quinze ou seize ans; une perspective d'allongement des études pour tous... La sociologie critique a désenchanté le modèle dans les années 1970 (Derouet, 1992). Dans les débats qui ont porté sur le caractère mystificateur de l'idéal d'égalité des chances, il est apparu que l'égalité ne constituait qu'une des traductions possibles de l'exigence de justice et que celle-ci était datée. La bourgeoisie, à la fin du XVIIle siècle, souhaitait mettre fin à un système où les classements sociaux reposaient sur la naissance et promouvoir de nouvelles hiérarchies fondées sur ce qu'on appelait à l'époque les talents. Née dans une conjoncture, cette définition de la justice peut disparaître, passer au second plan ou entrer en composition avec d'autres lorsque le contexte change. Au XIXe siècle, l'idéal d'égalité s'était imposé contre les droits des familles et l'intégration communautaire. Ces principes retrouvent une légitimité. Pour des raisons de réalisme économique, le projet d'égalité est toujours entré en compromis avec un principe d'efficacité. La mondialisation donne à celui-ci une nouvelle forme l'obligation de résultats dans un contexte de concurrence. D'autres références apparaissent: la reconnaissance des différences; les droits des usagers et la transparence du service public; les droits des enfants et des jeunes, etc. Cette pluralité de principes n'est pas nouvelle. La nouveauté réside dans le refus de toute réduction de cette complexité. Les acquis de la critique, les connaissances qu'elle a produites, mais aussi ses modes de pensée font partie du sens ordinaire des acteurs (Boltanski, 1990). Quelle que soit la définition du bien commun proposée, tous les arguments sont disponibles pour la critiquer: références philosophiques, statistiques, comparaisons internationales, etc.

Ainsi se définit le problème que doit résoudre la deuxième modernité: comment l'école peut-elle s'orienter dans cet univers à principes de justification multiple? C'est dans ces conditions que les établissements scolaires ont pris une place essentielle. S'il n'y a pas de compromis unique qui réaccorde le projet d'éducation à l'échelle nationale, voire internationale, il 
peut y avoir une série de compromis locaux, tous différents même s'ils s'inscrivent dans un cadre semblable.

La reformulation de la question de la justice amène une reformulation de la question de l'État. Les années 1970 sont passées d'une crise de l'Étatprovidence, considéré comme inefficace à la remise en cause de la légitimité même de l'intervention de l'État (Crozier, 1987). Les années 1980 ont donc vu l'État se retirer de certains terrains qu'il occupait. II peut s'agir dans certains cas d'un recul devant le marché. Dans d'autres, il s'agit plutôt d'un changement de forme. C'est tout l'enjeu de l'interprétation des démarches de III voie (Giddens, 1998). Le terme a été inventé par le néo-travaillisme britannique mais la démarche concerne tous les pays. Pour caractériser cet État, les sociologues français peuvent évoquer la philosophie du projet identifiée par Luc Boltanski et Eve Chiapello dans "Le nouvel esprit du capitalisme". Les anglais parlent plutôt d'un État managérial (Clarke \& Newman, 1997; Gewirtz, 2002) .

C'est à l'intérieur de ce cadre qu'il faut penser les rôles des écoles et des établissements scolaires dans le fonctionnement du système éducatif français dans les cinquante dernières années. Pour suivre ce parcours, il est essentiel de se déprendre d'un certain nombre d'idées reçues. La première concerne la centralisation de l'éducation en France. Celle-ci ne touche que certains aspects et certains secteurs. Les travaux des historiens montrent que la mise en système des établissements est récente. Elle ne remonte pas plus haut que les années 1960. Auparavant il existait plusieurs réseaux d'établissements où les statuts, les programmes, l'organisation des études et la formation des enseignants, pouvaient être très différents (Prost, 1984). La seconde concerne les rapports entre mise en système et égalité. La Ve République a unifié les établissements au nom de la rationalité mais aussi au nom de l'égalité des chances. Là aussi les travaux des historiens révèlent un paradoxe. Dans les années 1960 la diversité de l'offre d'éducation a permis une "démocratisation rampante" qui s'est arrêtée avec le début de la mise en système des années 1960 (Prost, 1985).

Le tableau va être dressé en trois grandes périodes correspondant à trois grandes lois. La loi de modernisation du système éducatif de 1975, la loi d'orientation de 1989, la loi d'orientation de 2005. Cette chronologie apparaît la plus commode et la plus claire même si elle fait passer au second plan un 
des enjeux essentiels des dernières années qui est l'intégration progressive de la France au système mondialisé. Dans les années 1980, la France a mis en œuvre les politiques compensatoires critiquées par le rapport $A$ nation at risk qui paraissait au même moment aux États-Unis. Ce décalage a été ensuite progressivement rattrapé. La Conférence de Lisbonne, en 2000, a intégré l'enseignement européen à un espace de concurrence mondial.

\section{Du début de la Ve République à la loi de modernisation du système éducatif de 1975: heurs et malheurs de la première modernité}

La Ve République est arrivée au pouvoir en 1958. Au plan scolaire, elle se trouvait confrontée à une série de non-décisions. II existe depuis la Première Guerre mondiale une tradition française qui prône la démocratisation des études par la création d'une école unique de 6 à 14 ou 15 ans (Garnier, 2008). Celle-ci a été stabilisée à la Libération par le Plan Langevin-Wallon. Les deux rédacteurs étaient proches du Parti Communiste et le Plan n'a jamais été appliqué mais il constitue une référence qui est peu à peu devenue mythique (Mialaret, 1997). Dans les années 1950 et 1960, cette orientation a convergé avec la perspective d'école compréhensive portée par l'OCDE. Dix-neuf projets ont été déposés sur le bureau de l'Assemblée nationale entre 1947 et 1958. Aucun n'a abouti. Une partie importante de la société - et pas seulement la droite conservatrice - craignait que l'ouverture à tous des études secondaires n'entraîne une baisse de leur niveau et la perte d'une tradition culturelle française. La Ve République a souhaité mettre fin aux atermoiements de la IVe. Le général de Gaule voyait dans l'école unique un instrument de l'unité nationale (Narbonne, 1994). Surtout, elle devait faire face à l'explosion scolaire (Cros, 1961), c'est-à-dire offrir un cadre pour scolariser les enfants du baby-boom. C'est dans cette conjoncture qu'elle a opté pour une mise en système qui a porté à son comble la tradition de centralisation. II y a là à la fois une conception de la justice et une conception de l'État. La conception de la justice s'appuie sur un sens élevé de l'intérêt général. Celuici traite les problèmes à partir de procédures impersonnelles et standardisées. II proscrit absolument tout ce qui serait acception de personne, arrangement en fonction des situations locales, etc. Le principal instrument de cette conception, la carte scolaire, permet d'anticiper la gestion des masses en affectant les enfants à l'établissement le plus proche de leur domicile. 
C'est dans ce cadre que s'est mise en place la longue marche vers le collège unique (Geminard, 1983). D'abord la prolongation de la scolarité obligatoire jusqu'à seize ans en 1959, puis le rassemblement des différentes filières dans un même établissement, le Collège d'Enseignement Secondaire (CES) en 1964. Les gouvernements du général de Gaulle n'ont pas été plus loin, à cause de dissensions internes. Georges Pompidou, premier ministre de 1962 à 1968, puis Président de la République de 1969 à 1974 était très attaché à la culture classique et craignait que le collège unique ne mettent fin à la transmission de ses valeurs. C'est sous la présidence de Valéry Giscard d'Estaing que la décision a été prise. La "Loi de modernisation du système éducatif" votée en 1975, est plus connue sous le nom de "réforme Haby", du nom du Ministre qui l'a portée. Elle correspond à la volonté de Valéry Giscard d'Estaing d'aligner la France sur les normes internationales. Cette décision arrivait un peu tard. La sociologie critique avait désenchanté les promesses de l'école unique en montrant que les inégalités n'étaient pas seulement liées aux dispositifs (filières séparées ou école unique) mais qu'il fallait aussi interroger les contenus, le langage professoral (Bourdieu \& Passeron, 1970) et ce que d'autres ont appelé plus tard le rapport aux savoirs (Charlot, Bautier \& Rochex, 1992). Une grande partie de l'opinion publique éclairée et, en particulier, les enseignants et les parents de classes moyennes ne pouvait pas adhérer au slogan que le ministre faisait diffuser par la télévision: "la même chance dans tous les cartables". En outre, et bien que Valéry Giscard d'Estaing s'inscrive dans une tradition libérale, la mise en œuvre de la loi de modernisation du système éducatif s'est située dans le prolongement de la conception de l'État gaulliste. La France a appliqué les programmes de l'école compréhensive plus tard que tous les autres pays de l'OCDE (mis à part l'Allemagne qui l'a refusée) mais avec une rigueur qui n'a existé nulle part ailleurs. La conception systématique, appuyée sur la carte scolaire a abouti à une organisation implacable. Cet excès a fait imploser le système. En 1980, un rapport de l'Inspection générale a montré que la loi n'était pas appliquée mais qu'en même temps ses principes désorganisaient le système éducatif. Dans la moitié des collèges, les principaux continuaient à composer des classes de niveau correspondant aux anciennes filières. En même temps les enseignants et les parents avaient perdu leurs références à cause de l'hétérogénéité des classes. 
Les polémiques qui ont entouré la réforme Haby risquent d'occulter des réalités plus profondes. La première est l'importance de la sociologie des organisations dans la réponse à l'échec de la réforme centralisée. Michel Crozier met en cause depuis 1964 le phénomène bureaucratique. L'impersonnalité de la règle tend au mépris des usagers; le pouvoir est accaparé par une corporation de fonctionnaires pour qui il est plus important d'obéir aux règles que d'obtenir des résultats, etc. Les analyses se fixent tout particulièrement sur l'Éducation nationale et développent la conviction qu'avec la croissance des effectifs cette machine est devenue ingouvernable. Le million de fonctionnaires a été atteint dans les années 1970: on parle à ce moment de la deuxième bureaucratie au monde après l'Armée rouge... Michel Crozier préconise une déconcentration centrée sur les établissements. Une de ses élèves, Dominique Paty a publié en 1980 une thèse "Douze collèges en France" qui montre en pleine période de centralisation la variété des établissements et l'effet de cette diversité sur la formation des élèves. Cette conviction surplombe l'ensemble des années 1980 à 2000. Elle concerne aussi bien les ministres de droite que les ministres de gauche. La formule de Claude Allègre, ministre de Lionel Jospin est restée célèbre: "dégraisser le mammouth". De la même manière tous les ministres ont eu pour objectif de diminuer le pouvoir de la corporation enseignante. II y a à cela à la fois des objectifs vertueux: donner du pouvoir aux usagers (parents et élèves) et des calculs de pouvoir. La Fédération de l'Éducation nationale (FEN) constitue une "forteresse enseignante" (Aubert, Bergounioux, Martin, Mouriaux, 1985). Cette puissance a causé sa perte. Même si la FEN constituait un allié de longue date du Parti Socialiste, le Ministre Alain Savary s'en est méfié. En 1985, il est apparu que la majorité de la FEN risquait d'échapper aux socialistes au profit d'autres partenaires de gauche. Le pouvoir socialiste a choisi de faire éclater la FEN.

Le successeur de René Haby, Christian Beullac, venait du monde de l'entreprise. Il a cherché une solution en s'appuyant sur sa culture managériale et a donc préparé une déconcentration centrée sur les unités de production, c'est-à-dire les établissements. Son projet s'appuyait sur une expérience menée dans l'académie de Toulouse sous l'impulsion de l'inspecteur général Maurice Vergnaud. Celle-ci avait abouti à une publication: "Cheminement vers l'autonomie" (1980). Les élections de 1981 ont fait que c'est la gauche qui a 
mis en œuvre cette politique d'autonomie des établissements. Elle lui a donné un sens politique qui correspondait à l'image qu'elle souhaitait afficher sans gommer les continuités: Maurice Vergnaud a été nommé Directeur des Collèges au ministère de l'Education nationale et son action s'est appuyée sur l'expertise de Dominique Paty, élève de Michel Crozier.

Une autre réalité apparaît: le désenchantement de l'idéal d'égalité des chances. Dans les années 1960, les jeunes et les familles ont constaté, et souvent à leur dépens que l'égalité d'accès n'est pas l'égalité de réussite. Les chercheurs commencent à distinguer massification et démocratisation (Merle, 2005). Surtout, la fin des années 1970 est marquée par un changement de stratégie des classes moyennes. Elles avaient soutenu le projet de collège unique dont elles attendaient l'accès de leurs enfants à l'école de la bourgeoisie depuis les années 1930. Elles ont obtenu ce qu'elles souhaitaient au cours des années 1960 et 1970. La loi de 1975 les a effrayées. Leur crainte est maintenant que leurs enfants soient noyés dans la masse. Leur revendication évolue donc. Comment trouver de nouvelles procédures de distinction dans un système en principe homogène? La solution la plus simple est le choix de l'établissement (Derouet, 2001). La carte scolaire reposait sur le principe que tous les établissements offraient le même service. Cette fiction a été dénoncée dès la fin des années 1970. En 1978, Le Figaro a publié un premier palmarès des Lycées. En 1982, le sociologue Robert Ballion identifie le phénomène dans un livre au titre prophétique "Les consommateurs d'école". Ce mouvement a surplombé le reste de la période: même si l'égalité des chances reste une figure obligée des rhétoriques politiques, la vraie question qui gouverne les politiques éducatives est l'autonomie de l'établissement, son sens politique et la liberté de choix des familles.

\section{De la loi de décentralisation de 1982 à la loi d'orientation de 1989: l'autonomie des établissements scolaires. Équité et démocratie de proximité}

L'année 1981 est marquée par le retour de la gauche au pouvoir après vingt-cing ans d'opposition. Celle-ci a à cœur de renouveler et même de renforcer les promesses de l'État-providence mais elle doit aussi tenir compte des acquis des sciences sociales. Aussi bien la mise en cause du phénomène bureaucratique par Crozier que la dénonciation de l'égalité des chances par 
Bourdieu. Elle ressent le besoin de changer ses méthodes et demande des rapports à différents spécialistes: Rapport De Peretti sur la formation des maitres; Rapport Legrand sur les collèges (1982); Rapport Prost sur les lycées; Rapport Favret sur l'enseignement primaire jusqu'à Bourdieu, principal auteur du rapport du Collège de France "Pour l'enseignement de l'avenir" (1985).

La conception de la justice évolue de l'égalité vers l'équité. Cela correspond aux recommandations de l'OCDE. L'égalité des chances apparaît à la fois formelle et utopique. Le relativisme impose de prendre en compte les situations, les personnes... et cette prise en compte peut aller jusqu'à la notion d'inégalités justes que le philosophe politique Rawls a introduit aux États Unis une dizaine d'années auparavant (1971). Au plan de l'État, la référence reste l'État-providence mais il y a plusieurs inflexions. Tout d'abord les moyens de la politique d'égalité des chances changent. La gauche tire les leçons de l'indifférence aux différences de l'école de la République. Les politiques scolaires restent fidèles au principe de l'égalité des chances mais elles cherchent de nouveaux moyens d'atteindre cet objectif en s'inspirant de la tradition anglo-saxonne du traitement de la pauvreté. L'égalité, ce n'est pas donner la même chose à tout le monde, c'est donner à chacun ce dont il a besoin. Ce qui peut impliquer des "inégalités justes". Pour donner à chacun ce dont il a besoin il faut le connaître. Cette orientation vers le local rencontre une autre proposition de la gauche. Celle-ci est inquiète d'une une crise du politique: les citoyens ne voient plus le lien entre ce qu'ils vivent et les débats qui se déroulent aux sommets de l'État. La gauche qui arrive au pouvoir se donne pour objectif de renouer ce lien par une démocratie de proximité.

C'est dans ce double contexte que s'inscrivent la création des ZEP et l'autonomie des établissements scolaires. Une loi générale de décentralisation a été votée en 1982.Le principal décret concernant l'éducation est paru en 1984: les établissements secondaires reçoivent le statut d'établissement public local d'enseignement (EPLE). La France reste fidèle aux programmes et aux règlements nationaux mais la notion de projet d'établissement devient centrale. II s'agit de trouver les adaptations pédagogiques qui permettent aux élèves de chaque établissement d'atteindre les objectifs nationaux.

Les premières années de la gauche sont marquées par une querelle concernant l'enseignement privé qui reprend le même chemin que la réforme 
Haby: une excessive volonté de standardisation va aboutir à l'affirmation du mouvement des consommateurs d'école. Le projet présidentiel de la gauche comprenait la réalisation d'un grand service public d'éducation incluant les établissements privés. La mise en œuvre de ce programme a déclenché une très importante protestation. II est vite apparu que ce n'était pas seulement la droite catholique qui s'opposait à cette mesure mais des familles de toutes opinions et de toutes conditions qui souhaitaient garder une possibilité de recours pour le cas où elles ne seraient pas d'accord avec les décisions de l'Éducation nationale (Langouet \& Leger, 1991). Cette opposition a triomphé dans des conditions dramatiques: le Président de la République a retiré le projet de loi inscrit à l'ordre du jour de l'Assemblée nationale.

Les années 1981-1982 sont celles du changement, voir de l'utopie. Un important remaniement ministériel en fin 1983 marque le retour au réalisme. L'éducation est directement touchée par la querelle de l'enseignement privé qui montre que la question du choix de l'établissement est devenue la principale question de l'éducation. Un autre changement est plus général. Même si la gauche se situe naturellement dans la tradition de l'État providence, les premiers effets de la mondialisation se font sentir: en 1983 faut-il sortir du serpent monétaire européen? François Mitterrand choisit de rester au sein des règles communautaires. A partir de là, se met en place la recherche d'un compromis. Le terme de Illème Voie a été revendiqué par le mouvement travailliste britannique. Le socialisme français est beaucoup plus discret et procède à une série de déplacements sous forme de thèse générale. La gauche veut proposer un nouveau contrat social, qui s'organise autour de la démocratie de proximité et de transparence du service public. On parle parfois de marché (cf. par exemple le dialogue entre Michel Rocard et Paul Ricœur publié en 1991 par la revue Esprit "Justice et marché") mais la France invente un compromis qui est le droit des usagers. L'usager est un client, qui évalue la qualité du service qui lui est rendu et revendique le droit de changer de fournisseur si le service ne lui convient pas. En même temps, c'est un citoyen qui demande la transparence du service public et une évaluation de l'efficacité des investissements qu'il consent au travers de l'impôt.

Même si le terme de III voie n'est pas prononcé, c'est bien une nouvelle forme de justice et une nouvelle forme de l'État. 
Pour les parents de classe moyenne la question du choix de l'établissement devient la revendication principale et aucun gouvernement ne peut se passer de leur soutien. La circulaire de Michel Rocard de 1989 confirme cette évolution et tente en même temps de l'encadrer. Le Premier Ministre fixe comme principal objectif à son gouvernement le renouvellement des rapports entre le service public et les usagers.

Pour le reste, la gauche poursuit la mise en place du projet de démocratisation de la première modernité. Le collège unique n'est pas stabilisé mais l'allongement des études se poursuit. En 1984, le Ministre Jean Pierre Chevènement a lancé un mot d'ordre qui a fait date: amener $80 \%$ d'une génération au niveau du baccalauréat. Cette évolution était annoncée par le rapport d'André Prost sur l'avenir des lycées. Après le collège unique, le mouvement de massification devait aboutir à un lycée pour (presque) tous, même si les filières préparent à des destins bien différents. Le mot d'ordre rencontre une attente de la société. Les années 1985-1995 sont marquées par une massification des lycées.

La Loi d'orientation sur l'éducation de 1989 propose une série de compromis entre les exigences contradictoires. Le titre même est significatif. La notion de système disparaît. L'État se borne à donner des orientations et laisse une liberté d'interprétation au local et en particulier aux établissements scolaires. Le préambule s'inscrit dans le prolongement de la première modernité même s'il tient compte des apports de la sociologie critique et affirme modestement que l'école "contribue dans la mesure de ses moyens à l'égalité des chances". La loi poursuit le mouvement d'extension de l'enseignement et d'allongement des études. Elle fixe pour objectif à l'école de donner une qualification à tous les enfants "vivant en France" (c'est-à-dire aussi aux jeunes issus de l'immigration) et d'amener $80 \%$ d'une génération au niveau du baccalauréat. En même temps, le préambule annonce une révolution copernicienne: l'objectif serait de "mettre l'élève au centre du système éducatif". Ce programme s'appuie sur une tradition pédagogique de l'Éducation nouvelle ("mettre l'enfant au centre des apprentissages") tout en la reformulant assez fortement: passer de l'enfant à l'élève est lourd de conséquence (Rayou, 2000). II entre en compromis avec les mouvements du droit des usagers et propose une nouvelle répartition du pouvoir: celui-ci passant de l'institution scolaire aux usagers. L'affirmation d'un tel principe 
constitue en soi une rupture dans l'histoire des politiques scolaires. La mise en œuvre a été très inférieure aux intentions affichées. Les années 1986-1989 ont été marquées par plusieurs grèves lycéennes que Lionel Jospin a traduites en demande de dignité. II a accordé des droits nouveaux aux lycéens: un droit d'association qui prend la forme de la création des Maisons des lycéens puis des Conseils de la vie lycéenne. Ces concessions ont permis de mettre fin honorablement aux grèves mais les élèves n'ont pas toujours habité les institutions dont ils avaient obtenu la création. Cela ne signifie pas que la demande de dignité n'existe pas (Merle, 2005) mais elle fait partie d'un ensemble plus complexe (refus de la forme scolaire, inquiétude sur l'avenir, etc. ) qui correspond à l'épuisement du modèle de la modernité.

Au plan pratique, la loi propose une régulation du système fondée sur la notion de contrat. Un contrat doit s'établir entre le projet de l'établissement et le projet des familles ou des élèves. Ceux-ci sont au départ faits pour s'entendre puisque l'objet du projet d'établissement est de tenir compte des caractéristiques du local et donc des familles dans la mise en œuvre des objectifs nationaux. Une question se pose cependant: "qu'est ce qui se passe si le projet d'une famille ne correspond pas au projet de l'établissement où la carte scolaire affecte son enfant?". La carte scolaire était contournée depuis longtemps par des familles avisées mais ce phénomène devait rester discret. À la suite de la loi de 1989, les Recteurs ont reçu des possibilités officielles d'assouplissement de la carte scolaire. Les usages ont été très différents selon les périodes et les académies mais le phénomène n'est plus clandestin.

La nouvelle organisation s'est mise en place au cours des années 1990. Elle a permis de faire face à une deuxième explosion scolaire qui a suivi le mot d'ordre d'amener $80 \%$ d'une génération au niveau du baccalauréat. Cette deuxième massification a été gérée grâce aux efforts des Régions mais elle n'a pas entrainé de changement véritable. Le collège unique qui constituait la pièce maîtresse du modèle de la première modernité ne sort pas de la crise (Dubet \& Duru-Bellat, 2002). Les rapports se succèdent sans grand effet. Au lycée, les moyens permettent d'assurer l'accueil des nouveaux élèves mais il n'y pas de réflexion sur les changements, pédagogiques, ou curriculaires qu'impliquerait l'accueil des nouveaux élèves. La seule véritable innovation, la création des baccalauréats professionnels, reste une opération administrative et n'est pas accompagnée d'une réflexion sur l'articulation entre 
culture classique et culture technique ni sur la manière dont celle-ci peut nourrir le projet de démocratisation. Sans vouloir nourrir les propos catastrophistes les années 1990 donnent bien l'impression de l'épuisement d'un modèle de démocratisation qui continue sur sa lancée mais qui a perdu son sens et ses bases sociales (Derouet, 1999).

\section{Les interrogations à la fin du XXe siècle: l'épuisement du modèle de la première modernité et la montée en puissance d'un modèle managérial porté par les organisations internationales}

La fin du $X X e$ siècle est donc marquée par un certain nombre d'interrogations qui marquent la fin du modèle de démocratisation de la première modernité. C'est sans doute ce qui explique la facilité avec laquelle la France s'est s'alignée sur les recommandations internationales qui mettent peu à peu en place un modèle correspondant à une deuxième modernité fondée sur l'obligation de résultats.

La rupture la plus visible, mais qui n'est pas la plus analysée, est l'échec de l'objectif fixé par la Loi de 1989 d'amener 80\% d'une génération au niveau du baccalauréat. Le mot d'ordre avait été lancé en 1984 et avait connu un incontestable succès. Les effectifs des lycées ont spectaculairement augmenté. Le taux de réussite au baccalauréat est passé de moins $40 \%$ d'une génération à plus de $60 \%$ en dix ans. Cette courbe a commencé à flotter en 1995 puis est légèrement retombée. Il est bien sûr possible d'évoquer la crise de l'emploi et le nombre de diplômés chômeurs ou considérablement déqualifiés mais l'essentiel est peut-être ailleurs. La coupure entre l'école et la vie sociale ordinaire avait été conçue au XVIIIE siècle comme une protection. II s'agissait de mettre la sélection scolaire à l'abri des pressions des Églises et des notables. Cette protection aboutit aujourd'hui à une perte de sens. Les élèves issus des nouvelles classes populaires ne retrouvent plus leurs enjeux dans ce monde aseptisé. II existe plusieurs façons de conceptualiser ces phénomènes. Bourdieu avait évoqué les capacités de mise à distance du langage professoral; Charlot, Bautier et Rochex ont travaillé sur les rapports aux savoirs. Ballion l'exprime autrement. II étudie les nouveaux lycéens qui sont obligés de faire des petits boulots pour payer leurs études. Ceux-ci sont sensibles au facteur d'inégalité que constitue ce travail. En même temps, ils 
disent que ce contact avec la "vrai vie" les aide à donner sens aux épreuves en papier de l'école. II est difficile de mesurer cette perte de sens mais il est incontestable qu'elle joue un rôle fondamental dans la crise du modèle de démocratisation de la première modernité. Ce n'est pas seulement l'idéal d'égalité des chances qui est en cause, c'est le "grand renfermement" qui isole l'école du monde ordinaire.

Cette perte de sens amène une partie de la gauche pédagogique à s'interroger sur l'allongement des études (Duru Bellat, 2006). Cet objectif faisait partie de la définition du bien de la première modernité. Un grand nombre d'étudiants s'entassent à l'université. Non seulement leur avenir, dans certaines filières apparaît incertain, mais ils ne semblent pas retirer de leur présence à l'université une véritable formation intellectuelle. L'impression générale est celle d'une perte de sens... En outre, cette augmentation du nombre des étudiants est évidemment très coûteuse. Elle amène une question: ces investissements ne seraient-ils pas plus utiles ailleurs, en particulier dans des interventions précoces contre l'échec scolaire?

C'est dans ce climat de désenchantement vis-à-vis de la première modernité que la France a été touchée par le changement de référentiel initié aux États-Unis dans les années 1980. La France a été influencée par les orientations portées par les organisations internationales mais elle a toujours interprété leurs recommandations en fonction de ses traditions nationales. La loi de 1975 et la mise en œuvre du collège unique constituent un excellent exemple. La France s'inscrit dans les orientations de l'OCDE concernant l'école compréhensive mais en même temps, elle se réfère à sa tradition d'école unique qui remonte aux compagnons de l'Université Nouvelle (1919) et au Plan Langevin-Wallon (1997). Dans les années 1980, le décalage a été patent. La France s'inspirait des expériences anglo-saxonnes de politique compensatoire qui remontaient aux années 1960. Aux Etats-Unis, le rapport A Nation at Risk développait une critique virulente de ces orientations et proposait une nouvelle définition du bien commun éducatif. Le but principal de l'éducation n'est pas l'égalité ou la cohésion sociale à l'intérieur d'une société donnée. C'est le maintien du rang du pays dans la compétition internationale. Une nouvelle conception de la justice s'est élaborée qui repose sur l'obligation de résultat. Chacun doit contribuer à sa manière à l'effort collectif et rendre compte de sa contribution. A partir de ce principe une instrumentation s'est 
mise en place qui sous tend une nouvelle régulation. Tout d'abord la définition des standards de compétence. II n'est pas possible de comparer les performances des systèmes éducatifs à partir des programmes nationaux. II faut trouver des notions transversales: c'est ainsi qu'émergent les standards de compétences, leurs définitions accompagne le mouvement Back to Basics. Le rapport $A$ Nation at Risk met en cause l'introduction de dimensions culturelles dans les apprentissages de base. L'ambition excessive des politiques démocrates a abouti à renforcer l'échec des enfants d'origine populaire. La formation de la masse est très importante car le rang du pays dans la compétition internationale dépend aussi de la qualité de sa main d'œuvre mais celle-ci doit être conçue à partir de compétences simples et principalement instrumentales. Cela ne signifie pas une baisse du niveau, car le même rapport préconise une élévation des standards qui constitue une constante aux États Unis. En même temps le rapport $A$ Nation at Risk appelle à un retour de l'intérêt pour la formation des élites. Ce sont les élites qui entraînent le reste du pays dans la compétition mondiale et leurs performances servent à tous. En cas de déclin économique, on sait que ce sont les personnes les moins formées qui payent le plus lourd tribut. A partir de là se met en place une régulation par benchmarking. Celle-ci peut aussi se réclamer d'une conception civique du bien commun: l'obligation de rendre compte. Cette thématique est très forte en France. Au delà de la critique de la centralisation, de la critique de l'État, il y a la critique d'une corporation de fonctionnaires qui a confisqué la définition de l'intérêt général et renvoie les revendications des usagers vers les intérêts particuliers.

Ce changement de référentiel caractérise une deuxième modernité qui gagne petit à petit du terrain au sein des organisations internationales. Pour l'Europe deux dates sont essentielles. La première porte sur la conception générale des politiques éducatives. La Conférence de Lisbonne de 2000 fixe un objectif: que l'Europe occupe le premier rang dans une économie de la connaissance en 2010. Pour atteindre ce but, les Etats européens doivent se rendre compte des performances de leur système éducatif. À partir de là se met en place un double système: une évaluation par compétences et des grandes enquêtes du type PISA qui permettent d'établir les comparaisons des performances et d'orienter les politiques en fonction des résultats (Derouet \& Normand, 2007). La seconde concerne un retour d'intérêt pour la formation 
des élites. C'est un des thémes centraux du rapport $A$ Nation at Risk. En déplaçant les investissements vers le soutien aux élèves en difficulté, les politiques démocrates ont failli compromettre le rand des États Unis dans la compétition internationale. Celui-ci dépend en effet en grande partie de la performance de ses élites. Cela signifie que l'essentiel de la partie se joue dans l'enseignement supérieur. Des classements des Universités, comme celui de Shanghai n'ont pu que renforcer ce mouvement, même s'ils ont été très discutés. En 1998, le ministre français Claude Allègre a lancé à la Sorbonne l'idée de la construction d'un espace européen de l'enseignement supérieur. Cette déclaration a ouvert la voie à ce qu'on appelle le processus de Bologne. La mise en place d'une architecture commune (le système LMD) permet aux étudiants de construire leurs diplômes à partir de crédits acquis dans différentes universités. Mais aussi la publication de références de qualité qui permettent de savoir si les différentes universités se situent au même niveau d'exigence.

Pour ce qui concerne les établissements scolaires, les recommandations internationales ont évolué des années 1980 aux années 2000. Un principe demeure: le progrès des systèmes éducatifs repose sur le progrès de chaque établissement. II s'agit ensuite de savoir comment obtenir ce progrès. Un mouvement de school efficiency a pensé obtenir cette amélioration par des incitations économiques: des primes pour les enseignants qui réussissent, la fermeture des établissements qui échouent, etc. Les limites de ce mouvement sont très vite apparues: la pression de l'évaluation entraîne la fuite des élèves et des enseignants. D'où une stratégie plus élaborée qui tente de passer par la conscience des personnes: le school improvement. II s'agit de soutenir le développement des établissements en s'appuyant sur un management participatif et un accompagnement du développement professionnel des enseignants.

Les premiers échecs de cette orientation internationale sont parvenus en France au moment où le mouvement d'autonomie des établissements initié en 1981, rencontrait ses limites. La politique du projet d'établissement a été vivante dans les années 1982-1995. Elle a amené de nombreux changements dans ce qu'on appelle en France la vie scolaire : la restauration, les pauses, les transports, etc. Elle n'a touché le domaine pédagogique qu'en des occasions exceptionnelles: le travail autonome dans les Centres de 
Documentation et d'Information (CDI), quelques opérations interdisciplinaires, quelques voyages, etc. Cette prudence était sans doute sage à l'origine. Pour faire accepter l'idée de projet d'établissement à des enseignants formés dans un système centralisé, il était sans doute sage de respecter leur domaine réservé. Vingt ans après, cette prudence devient un handicap. Prendre au sérieux la notion de projet d'établissement nécessite d'entrer dans les projets curriculaires. Cette démarche ne doit en aucun cas remettre en cause les programmes nationaux mais expliciter le travail d'adaptation local : l'Inspection générale constate que les programmes ne sont mis en œuvre qu'aux deux tiers. S'agit-il des deux mêmes tiers dans tous les établissements ? Faute d'entrer dans ces interrogations, à partir de 1995, les établissements continuent de faire des projets mais cette démarche devient une obligation administrative.

La crise du premier modèle de démocratisation est claire à la fin du $\mathrm{XX}^{\mathrm{e}}$ siècle. La France se trouve dans ce début de $\mathrm{XXI}^{\mathrm{e}}$ siècle avec plusieurs références de justice. La notion d'équité avait tenté une introduction maîtrisée de la différence. Ce compromis a très vite été dépassé. La quête de reconnaissance devient une définition de la justice à part entière. Cela entraîne une conséquence. L'échec scolaire et d'une manière plus générale le traitement de la pauvreté s'ethnicisent. Les pauvres ne sont pas seulement des pauvres, ce sont aussi des immigrés, souvent des musulmans (Lorcerie, 2003). La politique de redistribution doit composer avec une politique de reconnaissance dans ce que l'on appelle désormais une "égalité complexe".

En même temps les organisations internationales mettent au premier plan l'obligation de résultats et développe une instrumentation qui fait autorité.

\section{Égalité de résultats, choix de l'établissement et droit à l'expérimentation: une $\mathrm{III}^{\circ}$ voie libérales? La Loi d'Orientation et de programme pour l'avenir de l'école (2005).}

Les années 1995-2000 ont été marquées par l'entrée de l'école française dans un processus mondialisé d'évaluation et de régulation. Cette dimension est présente dans la Loi d'orientation de 2005 proposée par François Fillon. Toutefois, celle-ci ne constitue qu'une des pierres de l'édifice. 
Des changements essentiels relèvent d'une mesure plus générale, la Loi Organique relative aux Lois de Finance (LOLF) votée en 2001 à l'unanimité de l'Assemblée Nationale. Sa mise en œuvre a nécessité un long travail de préparation et c'est seulement en 2006 que le projet de Loi de Finance a été présenté selon ses principes. Le projet s'inscrit dans le prolongement du Nouveau Management Public. Le premier objectif de la LOLF est de rendre le budget de l'État plus lisible. Les finances ne sont plus présentées par nature des dépenses (fonctionnement, investissement, intervention ...) mais par politiques publiques (sécurité, culture, santé, justice...), désormais appelées "missions". Une mission est un ensemble de programmes concourant à une politique publique. Elle peut relever d'un ou plusieurs services, d'un ou plusieurs ministères. Un programme regroupe les crédits destinés à mettre en œuvre un ensemble cohérent d'actions correspondant à des objectifs précis. Les résultats attendus sont fixés dès l'origine et serviront de base à l'évaluation. Chaque programme est ainsi lié à un ou des projets annuels de performance (PAP) associés à la répartition prévisionnelle des emplois rémunérés par l'État avec présentation des actions, des coûts associés, des objectifs à atteindre, des résultats obtenus et des cibles pour les années à venir mesurés à l'aide d'indicateurs précis et choisis. Chaque PAP donne lieu à un rapport annuel de performance présenté au Parlement. Cette démarche de rationalisation des choix budgétaires est appuyée par la création d'un secrétariat d'État à la modernisation de l'État.

La Loi de finances de 2006 présente un système d'indicateurs qui permet de suivre les politiques académiques. Les Recteurs doivent présenter leurs dépenses par BOP (Budget Opérationnel). Les établissements ne sont pour le moment que faiblement concernés. Toutefois, le travail continue et les Recteurs mettent en place des politiques de contrat d'objectifs entre les établissements et les Académies qui reprennent les principes de la LOLF, en élaborant des indicateurs plus spécifiques. Les règles du Nouveau Management Public s'introduisent donc dans l'administration française sans soulever de véritables polémiques.

La mesure qui a le plus retenu l'attention du public dans la Loi d'Orientation de 2005 est l'engagement de l'État d'assurer à tous les enfants l'accès à un "socle commun de connaissances et de compétences". II s'agit d'un important changement de la définition de la justice. La Loi Jospin de 1989 
avait travaillé à partir de la notion d'équité, portée par l'OCDE. Celle-ci n'est pas abandonnée. Le principe de politique prioritaire pour les quartiers difficiles est conservé, même s'il est reformulé. Les politiques prioritaires des années 1980 considéraient l'échec scolaire comme un problème collectif et cherchaient à augmenter globalement la réussite des élèves dans les quartiers difficiles. La question a été reformulée dans les années 2000. II s'agit d'aider chaque enfant individuellement à construire son parcours dans un univers réticulaire. La notion de socle commun renvoie à une autre définition de la justice, travaillée par la Banque mondiale: le passage de l'égalité des chances à l'égalité de résultats à l'issue de la scolarité obligatoire. Cette orientation a été reprise par la Communauté européenne. En 2004, le programme "Éducation \& Formation 2010" a défini les compétences clés pour l'apprentissage tout au long de la vie. Claude Thelot a repris cette définition en France. La Loi de 2005 s'inscrit dans cette tradition mais elle tente d'adapter un compromis avec des traditions françaises antérieures. Pour les organisations internationales, la référence à la notion de compétence implique une rupture avec des programmes rédigés en termes de connaissance. Or la France est très attachée à ses programmes nationaux. La loi de 2005 parle d'un socle commun de connaissances et de compétences, sans expliquer l'articulation entre les deux. Cette lacune s'est bien sûr retrouvée au moment de la mise en œuvre. Le terme de socle commun tente d'établir une continuité avec le projet de culture commune formulé par le plan Langevin Wallon (Romian, 2000). II s'agissait, dans l'esprit de ses promoteurs, de renforcer la loi en l'appuyant sur un des fondements les plus légitimes de la gauche pédagogique. Les ambigüités sont telles qu'elles l'ont plutôt affaiblie. La notion de culture commune, fondement du programme de l'école unique, s'inscrit dans une conception humaniste qui va évidemment au delà des définitions instrumentales. Retrouve-t-on cette perspective dans la définition du socle commun? Quelques chercheurs de gauche ont hésité (Lelièvre, 2004). La majorité a situé le projet de socle commun dans le prolongement du "back to basics" prôné par le rapport $A$ Nation at Risk et a continué à revendiquer une culture commune plus large (Paget, 2006). Le compromis n'a donc pas pris. Tout au plus la notion de socle commun a t'elle été acceptée par un certain nombre côté responsables comme un moindre mal: elle peut fournir un point de repère dans le désordre actuel des collèges. 
La difficulté fondamentale se situe au delà des polémiques. Dans les recommandations internationales les compétences clés sont présentées comme un horizon souhaitable mais nul ne peut le garantir. Dans la Loi française, l'atteinte du socle commun correspond à un engagement de l'État. Cette différence apparaît très importante au moment où la Communauté européenne, qui a été à l'origine de ce mot d'ordre, s'interroge sur son réalisme. Les experts doutent que tous les élèves puissent réellement acquérir les compétences clés. Que faut-il faire dans ce cas? La réponse administrative est d'offrir aux jeunes qui n'ont pas saisi leur première chance des possibilités de retour en formation. Les limites de cette stagiérisation de la jeunesse en difficulté sont bien connues. Une autre solution serait de diminuer le niveau d'exigence du socle commun. Ce choix a été opéré par quelques pays anglosaxons qui concentrent leurs efforts sur la littératie et la numératie. Les nouveaux programmes de l'enseignement primaire, préparés par le successeur de François Fillon, Xavier Darcos, allaient dans ce sens. S'agissait-il d'une rupture ? Si c'est le cas, celle-ci n'a pas été clairement assumée et cette incertitude contribue à brouiller le paysage.

Dans ces conditions, il est difficile, quatre ans après le vote de la loi, de savoir quels changements elle a entrainés sur le terrain. Est-ce que les enseignants se sont approprié ses notions et ses objectifs?

En revanche, il apparaît peu à peu que la définition de la justice par l'égalité de résultats entraine un nouveau phasage des études. L'objectif d'égalité de résultats ne concerne que la scolarité obligatoire. L'enseignement supérieur évolue selon un autre principe, qui est la concurrence internationale. Cette évolution qui avait été annoncée par Claude Allègre en 1998, puis reprise lors de la Conférence de Bologne en 1999, se poursuit. Surtout, la Communauté européenne, qui avait été à l'origine écartée de ce processus, en a peu à peu pris le contrôle (Croché, 2009). Une troisième conférence, à Prague en 2001, a attiré l'attention sur la question de la certification de la qualité. Pour que les étudiants puissent construire des diplômes à partir de crédits obtenus dans différents universités, il faut que des instances extérieures garantissent la qualité de ces crédits. Toutes les universités ne jouent évidemment pas dans la même catégorie. II ne peut pas y avoir d'équivalences automatiques. Cette reformulation des enjeux ne se limite pas à des questions d'organisation. Elle entre dans la définition des contenus et de 
la "bonne science". Un premier modèle académique était fondé sur l'indépendance de la science par rapport au monde politique et économique. Le deuxième se définit au contraire par un fonctionnement en réseau où les agents politiques et économiques interviennent dans le fonctionnement de l'université, pour son financement mais aussi dans l'élaboration de sa politique scientifique (Gibbons, 1994).

En 2008, la Loi Liberté et Responsabilité des Université (LRU) a tiré les conséquences de ces évolutions. Elle a changé le management des universités, renforcé le pouvoir des Présidents afin d'augmenter l'efficacité dans un régime de benchmarking. Elle ouvre surtout des possibilités d'organisations en réseaux qui relient les universités au monde économique. Cette réforme a suscité de nombreuses protestations: deux mois de grève en 2009. Le gouvernement a tenu bon mais le malaise demeure. L'inquiétude des étudiants sur leur avenir converge avec le mécontentement des enseignants devant le renforcement des structures hiérarchiques. Au delà, un désajustement plus profond se fait jour. La loi LRU porte un modèle qui est celui de la deuxième modernité: la formation d'une élite performante au sein de réseaux internationaux. Le modèle de la première modernité, qui fait de l'allongement des études pour tous la définition du bien éducatif, reste présent aussi bien dans les discours politiques que chez les acteurs sociaux. Les universités se trouvent à l'heure actuelle dans une situation paradoxale. Elles doivent accueillir un afflux d'étudiants correspondant à l'idéal d'allongement des études. Même si ce mouvement est mis en cause il n'est pas prêt de cesser: il n'y a pas d'autre place pour un jeune dans la société que celle d'étudiant. En même temps, les universités doivent s'insérer et si possible conquérir un rang d'excellence dans un système international gouverné par la concurrence.

Autre désajustement. II manque un maillon pour assurer le passage entre un enseignement obligatoire dont l'objectif est l'égalité de résultats et un enseignement supérieur gouverné par la concurrence. Les lycées, qui assurent la scolarité des 15-18 ans n'ont pas été conçus dans cette optique. II faut donc envisager une réforme des lycées. Ceux-ci ne sont plus l'antichambre de la Cité savante mais la préparation à l'entrée dans un univers réticulaire gouverné par une philosophie de projet. Cette perspective était au cœur d'une réforme présentée par Xavier Darcos en 2008. Celle-ci a suscité 
une énorme protestation suivie d'une grève de lycéens. Le projet a été retiré à l'initiative du Président de la République. Enfin, la conjoncture se caractérise par un retour d'intérêt pour la question de l'orientation. II ne s'agit plus de l'orientation au sens du plan Langevin Wallon (repérer les aptitudes des enfants d'origine populaire) mais de la construction d'une compétence à l'orientation correspondant à l'organisation en réseau du monde : mobiliser des ressources autour d'un projet. C'est ce qui est présenté comme l'orientation active. Ce projet est soutenu par les entreprises et les collectivités territoriales.

Dans cette perspective managériale, l'unité de base de l'établissement scolaire est essentielle. La Loi de 2005 relance à sa manière l'idée de projet d'établissement avec l'article 34 qui ouvre des possibilités d'expérimentations pouvant aller jusqu'à la dérogation.

"Sous réserve de l'autorisation préalable des autorités académiques, le projet d'école ou d'établissement peut prévoir la réalisation d'expérimentations, pour une durée maximum de cinq ans, portant sur l'enseignement des disciplines, l'interdisciplinarité, l'organisation pédagogique de la classe, de l'école ou de l'établissement, la coopération avec les partenaires du système éducatif, les échanges ou le jumelage avec des établissements étrangers d'enseignement scolaire. Ces expérimentations font l'objet d'une évaluation annuelle. Le Haut Conseil de l'éducation établit chaque année un bilan des expérimentations menées en application du présent article"

Là aussi, cette mesure tente une synthèse entre deux orientations. II existe une tradition d'innovation de la gauche pédagogique (Groupe Français de l'Éducation Nouvelle; GFEN; Institut Coopératif de l'École Moderne; ICEMpédagogie Freinet; Centre d'Entrainement aux Méthodes d'Educations Actives; CEMEA etc ) qui travaille depuis les années 1930 à la recherche d'une pédagogie adaptée aux classes populaires. La philosophie du projet portée par le nouvel esprit du capitalisme développe une nouvelle conception de l'innovation: dans un univers en perpétuelle recomposition, le bon entrepreneur est celui qui sait mobiliser de nouveaux réseaux en fonction des évolutions de la conjoncture. C'est dans cet esprit que l'OCDE a commandé un rapport sur la manière dont l'éducation peut contribuer à construire une compétence à l'innovation. C'est dans le même esprit que la Communauté européenne a fait de 2009 l'année de l'innovation et de la créativité (Taddei, 2009). 
Les expérimentations article 34 sont nombreuses: il y aura bientôt un millier de projets parvenus à la mission qui suit cette opération à la Direction Générale des Enseignements Scolaires. Celle-ci récupère la tradition d'innovation des mouvements pédagogiques et promeut à la fois l'idée de diversification de l'offre d'éducation et l'idée de réforme par le bas.

Une deuxième mesure, qui n'est apparue qu'à la rentrée 2008, accentue les tendances. La carte scolaire a joué un rôle essentiel dans la mise en système des établissements et une certaine standardisation de l'enseignement. Les règles ont été plus ou moins assouplies depuis les années 1990 mais à la rentrée 2008, le Ministre Xavier Darcos a annoncé de nouvelles mesures d'assouplissement qui doivent aboutir à la suppression de la carte scolaire en 2010. Les Académies doivent publier un certain nombre d'indicateurs concernant les établissements et en particulier leurs performances. Le ministère publie en même temps une liste des motifs qui peuvent rendre légitime une demande de dérogation. C'est le triomphe du mouvement des consommateurs d'école et de la revendication des classes moyennes. C'est maintenant la question du choix de l'établissement qui est au centre du débat sur l'éducation. II est d'ailleurs significatif qu'aux élections présidentielles de 2008 la suppression de la carte scolaire ait figuré au programme des deux principaux candidats: le libéral Nicolas Sarkosy et la socialiste Ségolène Royal. Les argumentaires étaient différents. Pour Nicolas Sarkosy, il s'agissait d'un retrait de l'État d'un domaine où il n'a rien à faire. Pour Ségolène Royal, il s'agissait de renforcer les droits des usagers du service public. La convergence sur la décision est néanmoins significative. En même temps elle ne signifie nullement un triomphe du marché. De nombreuses régulations demeurent et la situation française reste très différente de celle de nombreux États voisins, la Belgique où le Royaume-Uni.

\section{Bilan et questions en 2009}

II est difficile de tirer des conclusions d'un tel parcours. II s'agit de processus en cours. Le but de l'analyse est plutôt de poser les bonnes questions, même si les réponses demanderont un certain temps.

Le parcours nous montre le passage d'un modèle d'État éducateur à un autre. Le premier modèle correspondait à l'État-providence avec une 
conception redistributive de la justice dont l'objectif était l'égalité des chances. La crise de ce modèle a amené différentes propositions de "Troisième voie" qui tentent de conserver certaines garanties de l'État-providence dans un univers à justifications multiples. Contrairement à certaines affirmations, il ne s'agit pas d'un recul de l'État devant le marché d'une recomposition de l'État autour de perspectives managériales. L'objectif d'égalité doit entrer dans des compromis avec la reconnaissance des différences et l'obligation de résultats.

Dans cette recherche de compromis, la place des établissements est centrale. Si tous les principes sont égaux au plan philosophique, tous ne sont pas également adaptés à une situation. La charge de la régulation passe donc du niveau national à celui des établissements. Le sens politique de cet intérêt pour le local a évolué au cours de la période. Dans les années 1980, les établissements étaient considérés comme des services publics de proximité au service de l'égalité des chances. Le consumérisme des classes moyennes s'est imposé peu à peu. Le local est maintenant devenu le lieu du choix de l'établissement. La question est aujourd'hui l'ambiguïté du mot d'ordre de diversification. S'agit-il simplement de faire place au marché? Les travaux des historiens ont montré qu'avant les années 1960 la diversité de forme des établissements a permis une démocratisation rampante qui s'est arrêtée lorsque la Ve République a commencé à les mettre en système (Prost 1989). Serait-il possible aujourd'hui de retrouver, dans un autre contexte, une diversification du service public qui permette aux enfants des classes populaires de construire le parcours qui corresponde à leurs enjeux?

De la même façon les analyses des difficultés du projet de démocratisation mettent en évidence le caractère ambigu du "grand renfermement" de l'école. Celui-ci a été conçu à l'origine comme une protection contre le pouvoir des nobles, du clergé, ensuite des patrons... Cette protection devient obstacle. Les enfants d'origine populaire ne retrouvent pas dans l'univers aseptisé de l'école les valeurs qui sous-tendent leur engagement dans le monde réel. Des propositions existent bien sûr de rapprochement de l'école et du monde économique. La formation tout au long de la vie prônée par la Communauté européenne met fin à la séparation entre le temps des études et le temps de la production. De même les Régions proposent une organisation réticulaire de la formation où les entreprises ont leur mot à dire sur le fonctionnement des établissements scolaires. II est facile 
de voir ce que le monde économique a à gagner à ce type de fonctionnement. Peut-il, comme il le prétend, ouvrir de nouvelles voies vers la démocratisation?

II est aussi possible de poser quelques questions plus profondes. Tout d'abord quelle est la réalité de l'apparence d'ordre construite par les enquêtes internationales et les indicateurs de performance ? Est ce que les enseignants adhérent à leurs principes? Saisissent-elles la réalité de la vie des classes et des établissements? Ou bien s'agit-il d'un filet très lâche qui produit une impression d'ordre tout en étant jeté sur un océan d'anomie? Que se passe-til derrière une apparente conformité et les séries de chiffres? II y a peu de protestations contre ce nouvel ordre et celles-ci ne mobilisent pas. II peut y avoir des formes de résistance plus sourde, des déplacements, etc. (DerouetBesson, 2007). Ceux-ci pour le moment n'apparaissent pas. Pourraient-ils un jour faire masse et proposer un nouveau modèle de démocratisation?

D'autre part les années 1980 - 2005 ont beaucoup parié sur les régulations locales. La crise financière de 2009 a amené une interrogation. Le local ne régule pas tout. II faut revenir sur la question de l'État. Enfin on peut se demander si les compromis actuellement portés par les organisations internationales ne courent pas le risque d'être dépassés. Ils laissent de côté des éléments importants de la réalité qui sont en train de se constituer en causes. Les mouvements pédagogiques parlent depuis longtemps des droits de l'enfant. La Loi d'orientation de 1989 a introduit le principe "mettre l'élève au centre du système éducatif". Même si la formule était prudente elle légitime un nouveau type de revendication: le respect du droit des élèves dans les établissements. De la même façon les propositions de régulation par benchmarking laissent de côté une importante question: est-ce que tous les pays sont à égalité dans la concurrence internationale? Les ressources, les équipements, les points d'appui ne sont évidemment pas semblables dans les pays du Nord et les pays du Sud. Ce sont de nouvelles définitions de la justice qui sont en train de se constituer.

\section{Referências}

AUBERT, Véronique; BERGOUNIOUX, Alain; MARTIN, Jean-Paul \& MOURIAUX, René (1985). La Forteresse Enseignante: La Fédération de l'Education Nationale. Paris: Fayard. 
BALLION, Robert (1982). Les Consommateurs d'École. Paris: Stock.

BALLION, Robert (1994). Les Lycéens et Leurs Petits Boulots. Paris: Hachette.

BOLTANSKI, Luc (1990). Sociologie critique et sociologie de la critique. Politix, $n^{\circ 10-11,}$ pp 124-134.

BOLTANSKI, Luc \& THEVENOT, Laurent (1991). De la Justification. Les Économies de la Grandeur Paris: Gallimard.

BOLTANSKI, Luc \& CHIAPELLO, Eve (1999). Le Nouvel Esprit du Capitalisme. Paris: Gallimard.

CHARLOT, Bernard; BAUTIER, Élisabeth \& ROCHEX, Yves (1992). École et Savoir dans les Banlieues et Ailleurs. Paris: Armand Colin.

CLARKE, John \& NEWMAN, Janet (1997). The Managerial State. London: Sage.

CROCHÉ, Sarah (2009). Bologne Confisqué. Constitution, autour de la Commission européenne, d'un acteur-réseau et d'un dispositif européen de l'enseignement supérieur. Thèse FUCAM - Université de Lyon.

CROS, Louis (1961). L'Explosion Scolaire. Paris: CUIP.

CROZIER, Michel (1963). Le Phénomène Bureaucratique Paris: Seuil.

CROZIER, Michel (1987). État Modeste, État Moderne. Paris: Fayard.

DEROUET, Jean-Louis (1992). École et Justice. De l'Égalité des Chances aux Compromis Locaux ? Paris: A.M Métailié.

DEROUET, Jean-Louis (2001). La sociologie des inégalités d'éducation à l'épreuve de la seconde explosion scolaire: déplacements des questionnements et relance de la critique. Éducation et Sociétés, Revue Internationale de Sociologie de l'Éducation, n5, pp 9-24

DEROUET, Jean-Louis (ed) (1999). L'École dans Plusieurs Mondes. Bruxelles: INRP/De Boeck.

DEROUET-BESSON, Marie-Claude (ed) (2007). Le quotidien du politique: ruse, souffrances et petits bonheurs. Éducation \& Sociétés Revue Internationale de Sociologie de l'Éducation, n¹9.

DEROUET, Jean-Louis \& DEROUET-BESSON, Marie-Claude (eds), (2009). Repenser la Justice dans le Domaine de l'Éducation et de la Formation. Genève : INRP/Peter Lang.

DEROUET, Jean-Louis; NORMAND, Romuald (eds) (2007). L'Europe de l'Éducation. Entre Management et Politique. Lyon : INRP/ESEN.

DUBET, François ; DURU-BELLAT, Marie (2002). L'Hypocrisie Scolaire. Pour un Collège enfin Démocratique. Paris: Le Seuil.

DURU-BELLAT, Marie (2006). L'Inflation Scolaire. Les Désillusions de la Méritocratie. Paris: Seuil.

GEMINARD, L (1983). Le Système Scolaire: Le Collège au Centre des Réformes. Paris: La Documentation Française.

GEWIRTZ, Sharon (2002). The Managerial School: Post-Welfarism and Social Justice in Education. London: Routledge. 
GEWIRTZ, Sharon (2009). Le nouveau Parti Travailliste et la Troisième Voie en éducation : tensions autour des Zones d'Action Éducatives. In Derouet, J-L \& Derouet-Besson, M-C, Repenser la Justice dans le Domaine de l'Éducation et de la Formation. INRP/Peter Lang,.

GIBBONS Michael (1994). The New Production of Knowledge. London: Sage.

GIDDENS, Anthony (1998). The Third Way: The Renewal of Social Democracy ("La troisième voie face: le renouveau de la social-démocratie"). Londres: Polity Press in association with Blackwell Publishers

Justice et marché (dialogue entre Michel Rocard et Paul Ricoeur) (1991). Esprit.

LANGOUET, Gabriel \& LEGER, Alain (1991). École Publique ou École Privée? Trajectoires et Réussites Scolaires. Paris: Éditions Fabert.

LEGRAND, Louis (1982). Pour un Collège Démocratique. Paris: La Documentation Française.

LELIÈVRE, Claude (2004). L'École Obligatoire : Pour Quoi Faires? Paris: Retz.

LORCERIE, Françoise (2003). L'École et le Défi Ethnique. Paris: ESF.

MERLE, Philippe (2005). L'Élève Humilié. L'École, un Espace de Non-droit. Paris : PUF. MIALARET, Gaston (1997). Le Plan Langevin-Wallon. Paris: PUF.

NARBONNE, Jacques (1994). De Gaulle et l'Éducation. Une Rencontre Manquée. Paris: Denoël.

PAGET, Denis (dir.) (2006). Aventure Commune et Savoirs Partagés. Paris: Syllepse.

PATY, Dominique (1980). 12 Collèges en France: le Fonctionnement Réel des Collèges Publics. Paris: La Documentation Française.

Pour l'enseignement de l'avenir. Rapport du Collège de France au Président de la République (1985). Paris, Collège de France.

PROST, Alain (1981). Histoire Générale de l'Enseignement et de l'Éducation en France. T.4: L'École et la Famille dans une Société en Mutation. Paris: Nouvelle Librairie de France.

PROST, Alain (1985). L'Enseignement s'est-il Démocratisé ? Paris: PUF.

Rapport de la National Commission on Excellence in Education (1983). A Nation at Risk: The Imperative For Educational Reform.

RAWLS, John (1971). A Theory of Justice. Oxford: Oxford University Press.

RAYOU, Patrick (2000) L'enfant au centre. Un lieu commun pédagogiquement correct. In J.-L. Derouet (ed), L'École dans Plusieurs Mondes. Bruxelles: INRP/De Boeck.

RICOEUR, Paul (2004). Parcours de la Reconnaissance. Paris: Stock.

ROMIAN Hélène (2000). Pour une Culture Commune. Paris: Hachette.

TADDEI, F (2009). Training Creative and Collaborative Knowledge-builers: a Major Challenge for 21st Century Education. Paris: OCDE.

VERGNAUD, Maurice (dir) (1980). Cheminements vers l'Autonomie. Toulouse: CRDP. 


\title{
O LUGAR DOS ESTABELECIMENTOS ESCOLARES EM FRANÇA NA V REPÚBLICA: UMA RECOMPOSIÇÃO PARALELA DAS FORMAS DA JUSTIÇA E DAS FORMAS DO ESTADO (1959-2009)
}

\begin{abstract}
Resumo
O artigo propõe um estudo do papel e do lugar dos estabelecimentos escolares nas três últimas Leis que regem o sistema educativo francês. O seu propósito é mostrar que aquelas Leis correspondem a uma recomposição paralela das formas da justiça e das formas do Estado. A Lei de modernisação do sistema educativo de 1975 tentava concretizar um objectivo de igualdade de oportunidades correspondente ao Estado-providência numa organização centralizada e mesmo autoritária. A Lei de orientação da educação de 1989 perseguiu o mesmo objectivo de igualdade mas pô-lo em acção num sistema desconcentrado cuja regulação assentava num contrato entre o projecto das famílias e o projecto dos estabelecimentos. A Lei de orientação e de programação de 2005 retoma essa preocupação de respeito dos direitos dos utentes e introduz uma obrigação de resutados correspondente a uma concepção gestionária do Estado.
\end{abstract}

Palavras-chave

Políticas de educação; Justiça; Estabelecimentos escolares; Sociologia do Estado 
THE PLACE OF SCHOOLS IN FRANCE AT V REPUBLIC: A PARALLEL RECOMPOSITION OF THE JUSTICE AND THE STATE FORMS

Abstract

This article proposes a study about the role and the place of schools when looking at the three last education Acts that lead french education system. Its goal is to argue that those Acts correspond to a parallel recomposition of the justice and the State forms. The education system modernisation Act of 1975 (Loi de modernisation du système éducatif) tries to built an objective of equality of chances that corresponds to an Welfare-state in a centralized even authoritarian organization. The orientation of education Act (Loi d'orientation de l'éducation) of 1989 prosecutes the same equality goal but put it on practice in a de-concentrated system whose regulation was supported by a contract between the families' project and the schools' project. The orientation and programmation Act (Loi d'orientation et de programmation) of 2005 reassumes that preoccupation about the respect of the users' rights and brings an obligation of results that corresponds to a managerial perspective of the State.

Keywords

Education policies; Justice; Schools; Sociology of the State

Recebido em Junho/2009

Aceite para publicação em Setembro/2009

Toda a correspondência relativa a este artigo deve ser enviada para: Jean Louis Derouet, UMR Éducation \& Politiques, Université Lumière Lyon 2, Institut National de Recherche Pédagogique, 19 allée de Fontenay, BP 17424, 69347 Lyon cédex 07- France. E-mail : jean-louis.derouet@inrp.fr 